This item was submitted to Loughborough's Research Repository by the author.

Items in Figshare are protected by copyright, with all rights reserved, unless otherwise indicated.

\title{
Optimization-based safety analysis of obstacle avoidance systems for
} unmanned aerial vehicles

PLEASE CITE THE PUBLISHED VERSION

http://link.springer.com/article/10.1007/s10846-011-9586-0?LI=true\#

\section{PUBLISHER}

(C) Springer Science+Business Media B.V.

\section{VERSION}

AM (Accepted Manuscript)

\section{LICENCE}

CC BY-NC-ND 4.0

\section{REPOSITORY RECORD}

Srikanthakumar, Sivaranjini, Cunjia Liu, and Wen-Hua Chen. 2012. "Optimization-based Safety Analysis of Obstacle Avoidance Systems for Unmanned Aerial Vehicles”. figshare. https://hdl.handle.net/2134/11136. 


\title{
Optimization-based Safety Analysis of Obstacle Avoidance Systems for Unmanned Aerial Vehicles
}

\author{
Sivaranjini Srikanthakumar, Cunjia Liu and Wen-Hua Chen \\ Department of Aeronautical and Automotive Engineering, Loughborough University, LE11 3TU, UK \\ sivaranjinisk@yahoo.co.uk, c.liu5@lboro.ac.uk and W.Chen@lboro.ac.uk
}

\begin{abstract}
The integration of Unmanned Aerial Vehicles (UAVs) in airspace requires new methods to certify collision avoidance systems. This paper presents a safety clearance process for obstacle avoidance systems, where worst case analysis is performed using simulation based optimization in the presence of all possible parameter variations. The clearance criterion for the UAV obstacle avoidance system is defined as the minimum distance from the aircraft to the obstacle during the collision avoidance maneuver. Local and global optimization based verification processes are developed to automatically search the worst combinations of the parameters and the worst-case distance between the UAV and an obstacle under all possible variations and uncertainties. Based on a 6 Degree of Freedom (6DoF) kinematic and dynamic model of a UAV, the path planning and collision avoidance algorithms are developed in 3D space. The artificial potential field method is chosen as a path planning and obstacle avoidance candidate technique for verification study as it is a simple and widely used method. Different optimization algorithms are applied and compared in terms of the reliability and efficiency.
\end{abstract}

Keywords - Clearance process, Obstacle avoidance, Optimization, Potential field method, Unmanned Aerial Vehicle.

\section{Introduction}

Due to the absence of a pilot, the use of UAVs has become increasingly popular in military and civilian applications. Path planning of UAVs with known and unknown obstacles is considered as one of the key enabling technologies in unmanned vehicle systems. Indeed, a significant amount of research has been devoted to this subject in recent years. In addition to offering better performance, the main industrial concern related to new methods is to reduce the risk of collision in the presence of all possible parameter variations and various failure conditions. Therefore, all proposed collision avoidance algorithms have to be verified under all operational conditions and variations that may be experienced during the life of the UAVs. The objective of this paper is to develop a process to support safety-critical obstacle avoidance systems for UAV operation. The certification process essentially aims at providing the evidence in order to certify that the aircraft is safe to fly in the presence of obstacles and parameter variations. This task is a very time consuming and expensive process, particularly for aircraft [1].

Without a pilot, computer algorithms must be developed to generate a feasible path in real time. Depending on the operation scenarios, there are different path planning methods. An UAV has to find a collision-free path between the departure and the destination (or a waypoint) configurations in a static and dynamic environment containing various obstacles. Several algorithms have been applied to path planning for UAV in the presence of known 
obstacles. Ray tracing and limit cycle navigation is combined in [2] for UAV operation in both 2D and 3D space, while Griffiths et al. present a rapidly exploring random tree (RRT) based path planner through 3D environment for an autonomous aerial vehicle [3]. In [4], both probabilistic roadmap-based and RRT algorithms are used for generating 3D collision free path for an autonomous helicopter. Bortoff develops a collision free path planning method using Voronoi graph search method [5], whereas a model predicative control based trajectory optimization method is used to avoid obstacles for Nap-of-the-Earth flight in [6]. UAV motion planning techniques based on potential field functions have been extensively studied; e.g. [7, 8]. UAV path planning using the artificial potential field method will be used as a candidate collision avoidance technique in this paper for safety assessment.

Three major concerns in regard to autonomous vehicle operation are efficiency, safety and accuracy. As the safety of autonomous vehicles is dependent on control systems and obstacle avoidance algorithms, it must be proven that the control systems and obstacle avoidance algorithms function correctly in the presence of all possible vehicle and environmental variations. Two particular difficulties faced by designers are a mismatching between the model used for algorithm development and the real vehicle dynamics, and various uncertainties in vehicle operations. To simplify the process of the algorithm development, in general a much simplified model that captures the main characters of a vehicle is used in the design stage under a number of assumptions or simplifications. This causes the mismatching between the model used in the design and real vehicle behavior. Furthermore, the variations of the autonomous vehicle dynamics in operation may arise due to the changes of the vehicle itself (e.g. the change of mass or the centre of gravity) or the change of the operational environment. Assessment of the safety must be performed not only on the nominal model, but also for all possible vehicle and environmental variations, and in the presence of the mismatching between the model used for the design and the real vehicle. Therefore, techniques and procedure are demanded to understand the behavior of UAVs in the presence of such uncertainties. They must cover all possible combinations of UAV parameters so that to guarantee that the worst-case performance is adequate, which is particularly important for safety critical functions such as collision avoidance.

Fault Tree Analysis method was applied to the TCAS (Traffic Alert and Collision Avoidance Systems) for UAVs safety analysis in [9], while Failure Modes and Effects Analysis (FMEA) was used [10]. In [11], Functional Failure Analysis (FFA) was performed for safety analysis of UAV operation including collision avoidance. Two critical hazards in UAV operation were defined in this analysis: midair collision and ground impact. Obstacle Analysis was applied to rotorcraft UAV in [12], where potential side effects and missing monitoring and control requirements were identified by step-by-step use of the obstacle analysis technique. In [13], the Markov Decision Process and Observable Markov Decision Process solvers was proposed to generate avoidance strategies optimizing a cost function that balances flightplan deviation with anti-collision. The performance of this collision avoidance system was evaluated using a simulation framework developed for TCAS studies. A framework for provably safe decentralized trajectory planning of multiple aircraft was presented in [14]. Each aircraft plans its trajectory individually using a receding horizon strategy based on mixed integer linear programming.

In this study, the minimum distance from the aircraft to an obstacle during a collision avoidance maneuver is chosen as the criterion for the performance assessment. To successfully perform collision avoidance maneuvers, the minimum distance to the obstacle $\left(d_{\text {min }}\right)$ must be greater than the radius of the obstacle $\left(r_{n}\right)$ including a safe margin. The worst 
case analysis in the presence of all the possible uncertainties is cast as a problem to find the combinations of the variations where the minimum distance to the obstacle $\left(d_{\text {min }}\right)$ appears. Instead of exhaustive searching the worst cases as in many practices, it is well known that optimization can effectively find a (local) minimum or maximum without evaluating all possible parameters in a solution space [15]. In this paper, the worst case analysis for collision avoidance algorithms is treated as a constrained nonlinear optimization problem with simulation being involved in each iteration. In order to pass the safety assessment of an anti-collision system, $d_{\min }$ in the worst cases must be greater than $r_{n}\left(d_{\min }>r_{n}\right)$ in the presence of all possible uncertain parameter variations. Otherwise, the obstacle avoidance algorithm and associated controllers have to be redesigned to satisfy the anti-collision specification. The proposed approach in this paper is applied to the collision avoidance algorithm using an artificial potential field method for a simple UAV model. However, the basic idea is applicable for other unmanned vehicles with collision avoidance algorithms developed by other methods. It shall be highlighted that it is not the intention of this paper to refine or develop a collision avoidance methods. Instead, it is to develop a new procedure to support the safety assessment of an existing collision avoidance algorithm for UAV operating in 3D environment.

The rest of the paper is organized as follows: the simplified kinematic and dynamic model of a 6 DOF UAV is introduced in Section 2. The clearance criterion of obstacle avoidance is also discussed in this section. Motion planning and collision avoidance algorithms in 3D environment are designed in Section 3 using the artificial potential field method. In Section 4, the obstacle avoidance algorithm is validated at nominal parameters. In Section 5, initial robustness analysis of the collision avoidance algorithm is carried out, where the optimization based verification process is introduced and local optimization algorithms are first presented. Two stochastic global optimization algorithm based verification processes are developed in Section 6. One is genetic algorithms (GA) and the other GLOBAL algorithm. Furthermore, in order to guarantee finding the worst-cases, a deterministic global optimization method, i.e. Dividing RECTangles (DIRECT), is applied to the worst case analysis of the collision avoidance algorithm in Section 7. Simulation results are presented to verify the proposed verification processes. Finally, Section 8 concludes the paper and outlines future research directions.

\section{UAV MODEL AND CLEARANCE CRITERION}

\section{A. UAV Model}

In order to present a clearance criterion of obstacle avoidance systems, an UAV model is considered for the algorithm development and assessment. A six-degree-of-freedom (6DOF) rigid-body aircraft model is considered in this study. The configuration vector $s=(x, y, z, \phi$, $\theta, \psi)$ is used to specify the position and orientation of the UAV in the global coordination, where $q_{0}=(x, y, z)$ is the c.g. (center of gravity) position of the vehicle and $\boldsymbol{\varphi}=(\phi, \theta, \psi)$ are the Euler angles, with $\phi$ as the roll, $\theta$ as the pitch, and $\psi$ as the yaw. 
The kinematic model is given by the following two equations [7], [8]:

$$
\begin{gathered}
{\left[\begin{array}{c}
\dot{x} \\
\dot{y} \\
\dot{z}
\end{array}\right]=\left[\begin{array}{ccc}
c_{\psi} c_{\theta} & c_{\psi} s_{\theta} s_{\phi}-s_{\psi} c_{\phi} & s_{\psi} s_{\phi}+c_{\psi} c_{\phi} s_{\theta} \\
s_{\psi} c_{\theta} & c_{\psi} c_{\phi}+s_{\phi} s_{\theta} s_{\psi} & s_{\theta} s_{\psi} c_{\phi}-c_{\psi} s_{\phi} \\
-s_{\theta} & c_{\theta} s_{\phi} & c_{\theta} c_{\phi}
\end{array}\right] v_{1}} \\
{\left[\begin{array}{c}
\dot{\phi} \\
\dot{\theta} \\
\dot{\psi}
\end{array}\right]=\left[\begin{array}{ccc}
1 & s_{\phi} t_{\theta} & c_{\phi} t_{\theta} \\
0 & c_{\phi} & -s_{\phi} \\
0 & s_{\phi} / c_{\theta} & c_{\phi} / c_{\theta}
\end{array}\right] v_{2}}
\end{gathered}
$$

where velocities are described in a body fixed frame with liner velocity $v_{l}=\left[\begin{array}{lll}u & v & w\end{array}\right]^{T}$ and angular velocity $v_{2}=\left[\begin{array}{lll}p & q & r\end{array}\right]^{T}$. Furthermore, the following notations are used: $s . \equiv \sin (),. c . \equiv$ $\cos (),. t . \equiv \tan ($.$) .$

The longitudinal and lateral dynamics for a fixed wing aircraft are given by the linear equations [18]

$$
\begin{gathered}
\dot{u}=X_{u} u+X_{w} w+X_{q} q+X_{\theta} \theta+X_{\delta_{e}} \delta_{e}+X_{t} \tau \\
\dot{w}=Z_{u} u+Z_{W} w+Z_{q} q+Z_{\theta} \theta+Z_{\delta_{e}} \delta_{e}+Z_{t} \tau \\
\dot{q}=M_{u} u+M_{w} w+M_{q} q+M_{\theta} \theta+M_{\delta_{e}} \delta_{e}+M_{t} \tau \\
\dot{v}=Y_{v} v+Y_{p} p-\left(u_{0}-Y_{r}\right) r+Y_{\phi} \phi+Y_{\psi} \psi+Y_{\delta_{a}} \delta_{a}+Y_{\delta_{r}} \delta_{r} \\
\dot{p}=L_{v} v+L_{p} p+L_{r} r+L_{\delta_{a}} \delta_{a}+L_{\delta_{r}} \delta_{r} \\
\dot{r}=N_{v} v+N_{p} p+N_{r} r+N_{\delta_{a}} \delta_{a}+N_{\delta_{r}} \delta_{r}
\end{gathered}
$$

where $\delta_{\text {con }}=\left[\boldsymbol{\delta}_{\mathrm{e}}, \boldsymbol{\delta}_{\mathrm{a}}, \boldsymbol{\delta}_{\mathrm{r}}, \tau\right]^{\mathrm{T}}$ are control inputs, corresponding to the elevator, aileron, rudder deflection angles, and thrust, respectively. The stability and control derivatives used in this dynamic model are derived from a nonlinear UAV model using linearization. Therefore, these derivatives depend on the physical parameters and aerodynamic coefficients of the UAV. Several derivatives are of particular interest in this study and used as uncertainty parameters, which are $X_{u}, X_{w}, Z_{u}, Z_{w}, X_{t}, Z_{\delta e}, Y_{v}, Y_{\delta a}, Y_{\delta r}$ being inversely proportional to the aircraft mass $(m), X_{t}$ and $M_{\delta e}$ proportional to aerodynamic coefficients of $C_{\delta t}$ and $C_{m \delta e}$ respectively.

\section{B. Clearance Criterion}

An UAV has to find a collision-free path between the starting pointing and the goal (e.g. waypoint) in an environment containing various static obstacles. Specifically, spherical obstacles are considered in this study but it is applicable to other obstacles. To assess the safety of UAVs, the minimum distance to the obstacle $\left(d_{\min }\right)$ is defined as the clearance criterion in the time domain. In order to maintain safety, as shown in Fig.1, the influence range of an obstacle is determined from the radius of the obstacle plus a specified safe margin. The specified safe margin can be chosen according to the UAV's dimensions. As mentioned above, the artificial potential field method is used in developing obstacle avoidance algorithms in this paper. In this framework, obstacle avoidance maneuver can be 
performed within the repulsive potential field influence range of an obstacle, where the UAV is repulsed from the obstacle and attracted to its goal position.

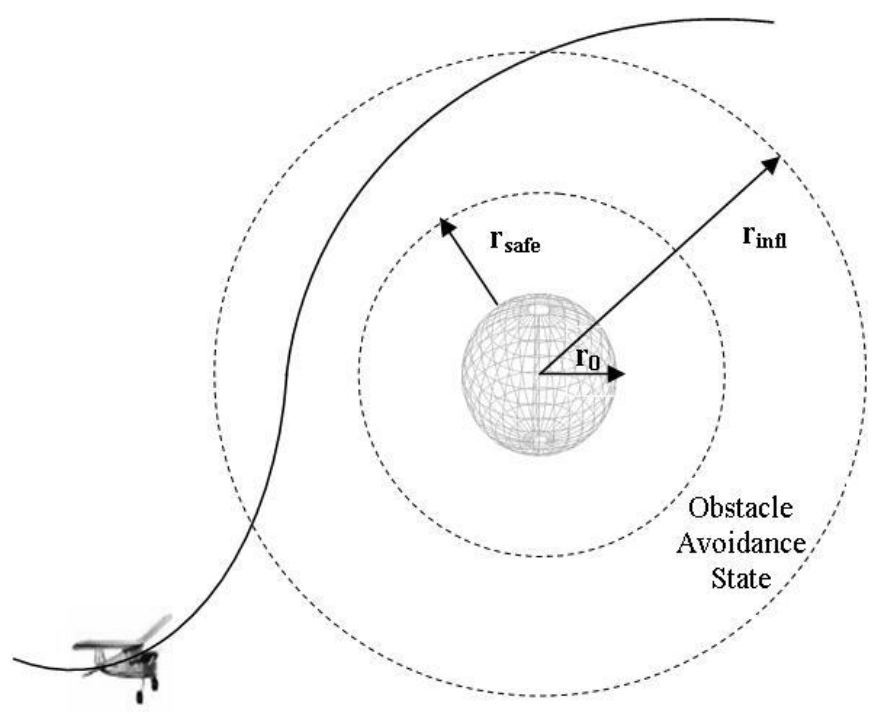

Fig.1. Obstacle avoidance clearance criterion

For a spherical obstacle, the influence range is chosen as the radius of $r_{\text {infl }}$ which is greater than the radius of the obstacle $\left(r_{0}\right)$ and the safe margin $\left(r_{\text {safe }}\right)$. Letting $r_{n}=r_{0}+r_{\text {safe }}$, the anticollision condition is defined as $d_{\min }>r_{n}$. In the obstacle avoidance clearance process, all violations of these clearance criteria must be found and the worst-case result for each criterion computed. The corresponding worst-case combination of uncertainties must also be computed.

\section{MOTION CONTROLAND OBSTACLE AVOIDANCE}

Fig. 2 provides an overview of the motion planning and control architecture [16]. The goal of motion planning is to generate a desired trajectory so that the UAV can track. Aircraft longitudinal and lateral dynamics and kinematic equations are considered for the clearance process. The high level mission planer usually supplies waypoint information to the motion controller. Then the motion planner retrieves the waypoints and generates a desired trajectory. The inner-loop control law is responsible to compute the input signals that drive the motors and control surfaces to force the UAV to fly at a desired linear velocity and attitude so the collision avoidance path generated by the motion controller can be followed.

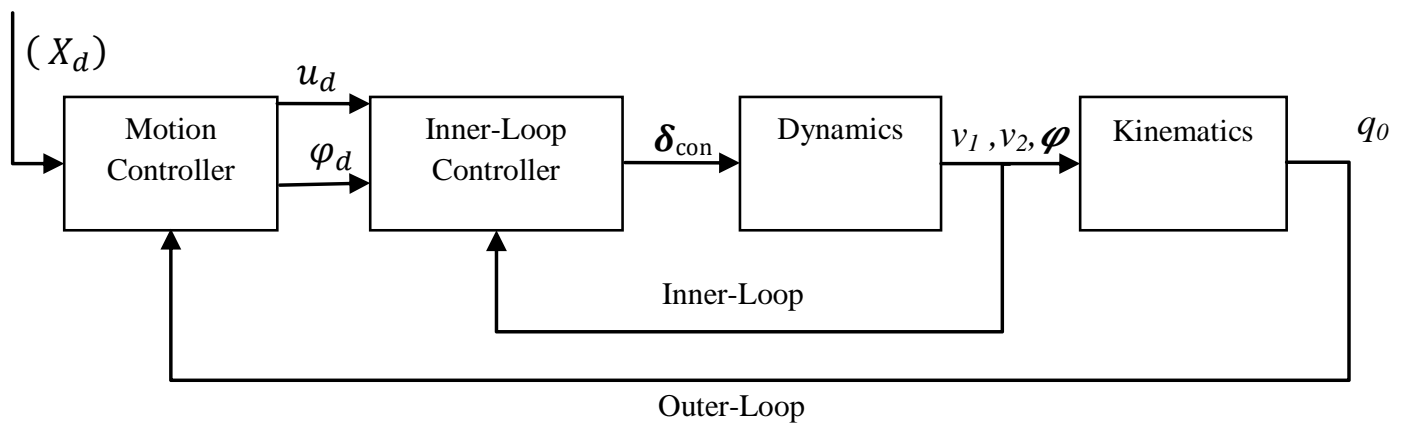

Fig.2. Motion planning and control structure 


\section{A. Motion Controller Using Potential Field Method}

The potential field method was first used by Khatib for manipulators and mobile robots path planning in the 1980s [19]. The basic concept of the potential field method is to fill the robot's workspace with an artificial potential field in which the robot is attracted to its goal position and repulsed away from any obstacles. The UAV path planning is, in a sense, similar to that of a mobile robot. The combination of the attractive force to the goal and repulsive forces away from any obstacles drives the UAV in a safe path to the goal.

Let $q_{0}=(x, y, z)$ denote the current UAV point in airspace. The usual choice for the attractive potential is the standard parabolic that grows quadratically with the distance to the goal, such that

$$
U_{\text {att }}\left(q_{0}\right)=\frac{1}{2} k_{a} d_{\text {goal }}^{2}\left(q_{0}\right)
$$

where $d_{\text {goal }}=\left\|q_{0}-X_{\text {goal }}\right\|$ is the Euclidean distance of the UAV's current position $q_{0}$ to the goal $X_{\text {goal }}$ and $k_{a}$ is a scaling factor $[17,20]$. The gradient is calculated as

$$
\nabla U_{a t t}\left(q_{0}\right)=k_{a}\left(q_{0}-X_{\text {goal }}\right)
$$

The attractive force considered in the potential field based approach is the negative gradient of the attractive potential

$$
F_{a t t}\left(q_{0}\right)=-\nabla U_{a t t}\left(q_{0}\right)=-k_{a}\left(q_{0}-X_{\text {goal }}\right)
$$

By setting the vehicle velocity vector proportional to the vector field force, the force $F_{\text {att }}\left(q_{0}\right)$ drives the UAV to the goal with a velocity that decreases when the UAV approaches the goal.

The repulsive potential keeps the vehicle away from obstacles. This repulsive potential is stronger when the UAV is closer to the obstacles and has a decreasing influence when the UAV is far away. A possible repulsive potential generated by obstacle $i$ is

$$
U_{\text {rep }_{i}}\left(q_{0}\right)= \begin{cases}\frac{1}{2} k_{\text {rep }}\left(\frac{1}{d_{\text {obst }}\left(q_{0}\right)}-\frac{1}{d_{0}}\right)^{2}, & \text { if } d_{\text {obst }_{i}}\left(q_{0}\right) \leq d_{0} \\ 0, & \text { if } d_{\text {obst }_{i}}\left(q_{0}\right)>d_{0}\end{cases}
$$

where $i$ is the number of the obstacle close to the UAV, $d_{o b s t i}\left(q_{0}\right)$ is the closest distance to the obstacle $i, k_{r}$ is a scaling constant and $d_{0}$ is the obstacle influence threshold. The negative gradient of the repulsive potential, $F_{\text {repi }}\left(q_{0}\right)=-\nabla U_{\text {repi }}\left(q_{0}\right)$, is given by,

$$
F_{\text {rep }_{i}}\left(q_{0}\right)= \begin{cases}k_{r}\left(\frac{1}{d_{\text {obst }_{i}}\left(q_{0}\right)}-\frac{1}{d_{o}}\right) \frac{1}{d_{\text {obst }}^{2}\left(q_{0}\right)} \hat{e}_{i}, \text { if } d_{\text {obst }_{i}}\left(q_{0}\right) \leq d_{o} \\ 0, & \text { if } d_{\text {obst }_{i}}\left(q_{0}\right)>d_{o}\end{cases}
$$

where $\hat{e}_{i}=\frac{\partial d_{o b s t}\left(q_{0}\right)}{\partial\left(q_{0}\right)}$ is a unit vector that indicates the direction of the repulsive force [21]. Therefore,

$$
\left[\begin{array}{c}
\dot{x}_{d} \\
\dot{y}_{d} \\
\dot{z}_{d}
\end{array}\right]=-\nabla\left(U_{a t t}\left(q_{0}\right)+U_{r e p}\left(q_{0}\right)\right)=\left(F_{a t t}\left(q_{0}\right)+F_{r e p_{i}}\left(q_{0}\right)\right)
$$


After the desired global velocity is calculated by the potential field method, the corresponding desired linear velocity $u_{d}$ and attitude $\boldsymbol{\varphi}_{d}=\left(\phi_{d}, \theta_{d}, \psi_{d}\right)$ can also be obtained based on UAV's kinematic model using the following equations:

$$
\begin{gathered}
u_{d}=k_{u}\left(\sqrt{\dot{x}_{d}^{2}+\dot{y}_{d}^{2}+\dot{z}_{d}^{2}}\right) \\
\phi_{d}=0 \\
\theta_{d}=\operatorname{atan} 2\left(-\dot{z}_{d}, \sqrt{\dot{x}_{d}^{2}+\dot{y}_{d}^{2}}\right) \\
\psi_{d}=\operatorname{atan2}\left(\dot{y}_{d}, \dot{x}_{d}\right)
\end{gathered}
$$

where gain $k_{u}$ is introduced to allow for additional freedom in weighting the velocity commands. The pitch and yaw angle guidance laws are designed so that the vehicle's longitudinal axis steers to align with the gradient of the potential field. The roll angle guidance law is designed to maintain the level flight.

\section{B. Inner-Loop Controller}

To accomplish the goal of driving the UAV flying at the desired linear velocity $u_{d}$ and desired attitude angles $\boldsymbol{\varphi}_{d}$, the first step is to compute the error between the true linear, the attitude angles and the desired ones, respectively. To this effect, let $\mathrm{e}_{\mathrm{u}}=\left(u_{d}-u\right), e_{\delta a}=\left(\phi_{d}-\phi\right), e_{\delta e}$ $=\left(\theta_{d}-\theta\right)$ and $e_{\delta r}=\left(\psi_{d}-\psi\right)$ denote the linear velocity and attitude angle errors, respectively. A simple PID control law is proposed as

$$
\delta_{P I D}=K_{p} e+K_{i} \int_{o}^{t} e(\tau) d \tau+K_{d} \frac{d e}{d t}
$$

Four PID controllers are designed for controlling linear velocity and three attitude angles, respectively. As the angular rates $p, q$ and $\mathrm{r}$ are available in flight control, the corresponding derivative terms in the PID controllers are replaced by their angular rate feedback.

\section{COLLISION AVOIDANCE VALIDATION AT NOMINAL CASE}

In this section, the proposed collision avoidance algorithm and controller are validated at the nominal parameters. The simulation results for a UAV approaching a spherical obstacle are presented at the nominal parameters. The nominal parameter values are $m=1.9 \mathrm{~kg}, C_{m \delta e}=-$ 1.13 , and $C_{\delta t}=12.19$. The initial linear and angular velocity vectors are $(15,0,0) \mathrm{m} / \mathrm{s}$ and $(0$, $0,0) \mathrm{rad} / \mathrm{s}$ for the nominal case. The initial Euler angle is $(0,0,0.9) \mathrm{rad}$. Safe margin is chosen as $5 \mathrm{~m}$. The PID controller gains and motion planner parameters for potential field force are also tuned and set to fixed values for the verification process.

In the simulation, the initial departure point is $(0,0,20) \mathrm{m}$, and the spherical obstacle is located at $(250,250,-10) \mathrm{m}$ with a radius $r_{0}$ of $20 \mathrm{~m}$. Therefore, the safety radius is $25 \mathrm{~m}$ including safe margin. The simulation result at the nominal parameters is shown in Fig.3. The 
minimum distance to the obstacle is obtained as $29.6166 \mathrm{~m}$ which is greater than obstacle safety radius $25 m\left(d_{\min }>r_{n}\right)$. This concludes that the obstacle avoidance algorithm works correctly at the nominal parameters. We now verify the proposed obstacle avoidance algorithms using the proposed optimization based clearance approach in 3D environment.

\section{Obstacle avoidance at nominal parameters}

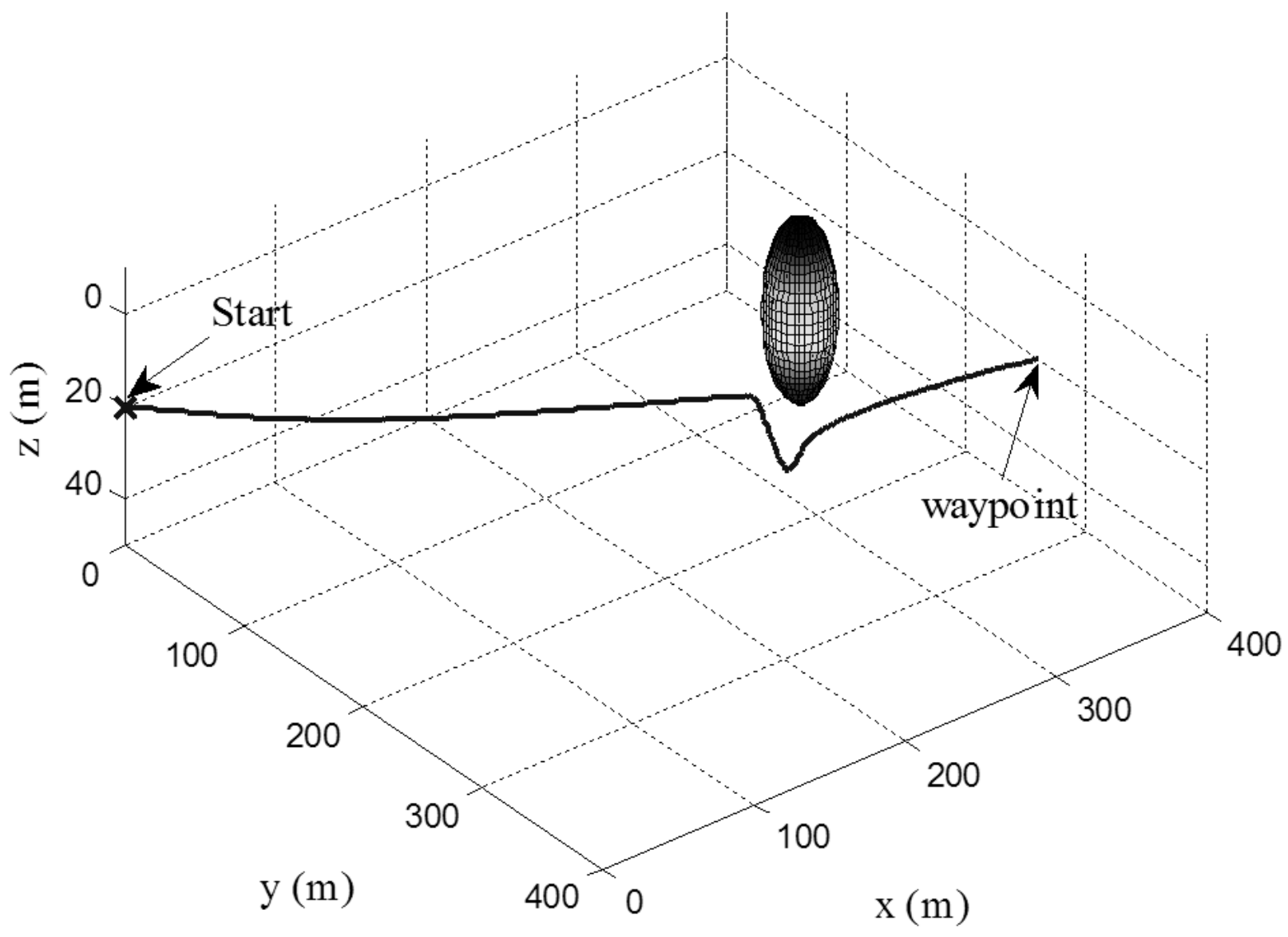

Fig. 3. Simulation result for UAV collision avoidance at nominal parameters

\section{Initial Robustness Analysis and Local Optimization Method}

Initial robustness analysis of the proposed algorithm is carried out in this section. Uncertainties are considered in the dynamic model (mass and two aerodynamic coefficients), and each uncertain parameter is allowed to vary within $\pm(10$ or 20$) \%$ of its nominal value. These are firstly considered within lower and upper bounds, i.e. $m=[1.52,2.28] \mathrm{kg}, C_{m \delta e}=[-$ $1.243,-1.017]$ and $C_{\delta t}=[10.971,13.409]$. For the purpose of comparison, the uncertain parameters are normalized to have a variation within the range. Fig. 4 shows variations of the minimum distance to the obstacle with respect to the normalized uncertain parameters of mass, $C_{m \delta e}$ and $C_{\delta t}$. There is a significant variation in the distance with the variations of these 
uncertain parameters. The minimum distance to the obstacle monotonically decreases with the increase of the $m$ and $C_{m \delta e}$, while $d_{\min }$ increases with the increase of $C_{\delta t}$.

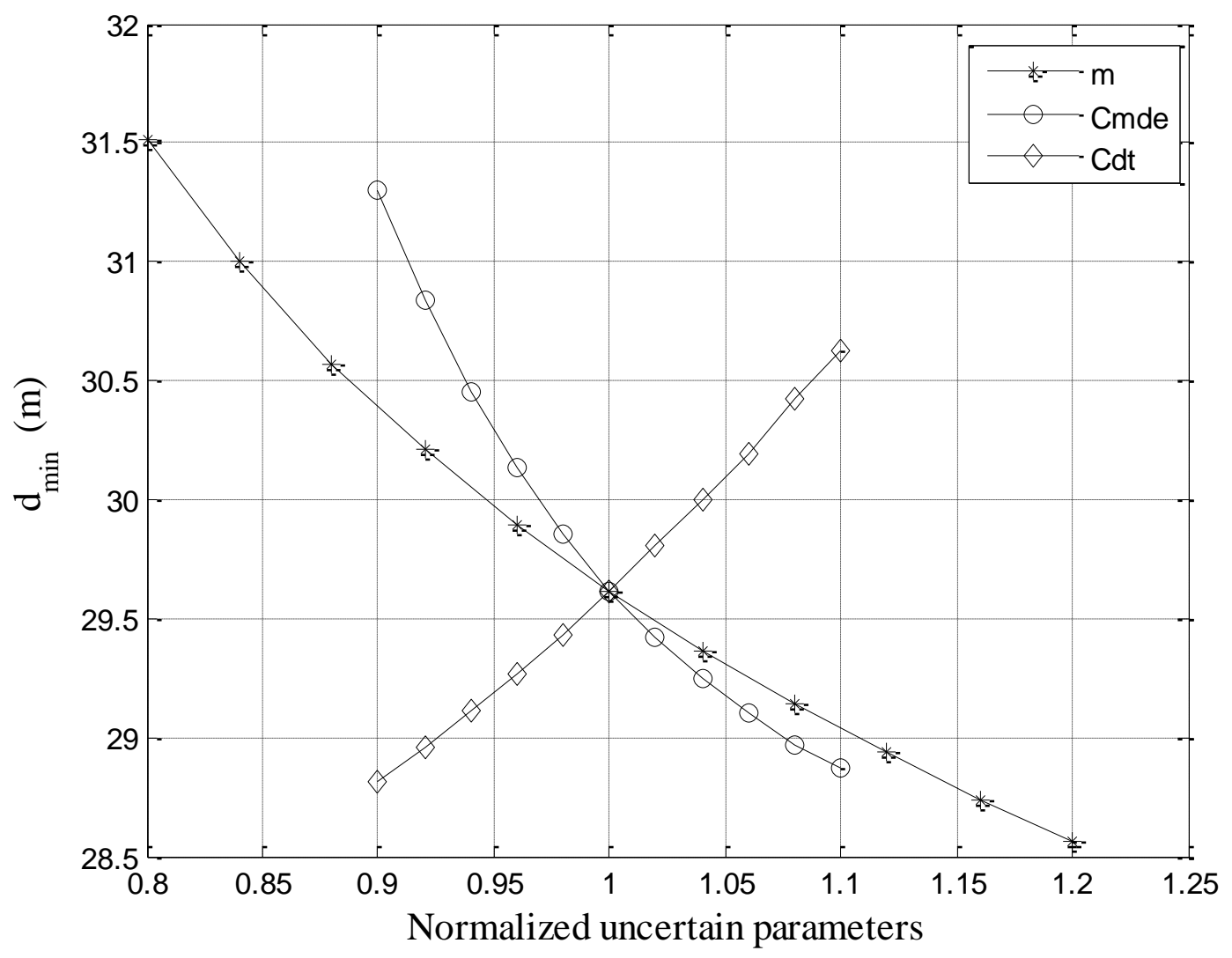

Fig.4. Mass, $\mathrm{C}_{\mathrm{m} \delta \mathrm{e}}$ and $\mathrm{C}_{\delta \mathrm{t}}$ variations

\section{A. Optimization-based Worst-Case Analysis}

In this paper, the optimization clearance process is applied to the UAV obstacle avoidance systems. If the minimum distance to the obstacle is greater than a safety radius of an obstacle $\left(d_{\min }>r_{n}\right)$ during the UAV moving, the proposed anti-collision algorithm is safe. When the optimization clearance process is applied to the system, this anti-collision condition is checked for all possible variations. The local and global optimization methods are applied to the problem of finding a worst-case combination of the condition and parameters for the UAV collision avoidance systems. Uncertain parameters are considered that lies between given upper and lower bounds. It shall be highlighted that the collision avoidance algorithm is developed only based on the kinematic model, but it has to be implemented through aircraft dynamics as described to drive the aircraft to follow the desired total speed and attitude. There is a mismatching in the model structure and complexity. Furthermore, the parameters in the inner-loop controllers also have a significant influence in collision avoidance. This problem caused by the structural uncertainty is unlikely solved by formal methods. 
Simulation based optimization is proposed in this paper. The objective function is defined as

$$
\begin{gathered}
d_{\text {min }}=\min (d(t)) \quad \text { for } t \leq T(\mathrm{sec}) \\
\text { s.t } \quad P_{L} \leq P \leq P_{U}
\end{gathered}
$$

where $P$ is the uncertain parameters set. $P_{L}$ and $P_{U}$ are the lower and upper bounds of $P, d(t)$ is the distance to the obstacle, $T$ is the collision avoidance maneuver during the period and $d_{\min }$ is the minimum distance to the obstacle.

\section{B. Local Optimization-based Worst-Case Analysis}

Sequential Quadratic Programming (SQP) method is a standard general purpose algorithm for solving smooth and well-scaled nonlinear optimization problems when functions and gradients can be evaluated with high precision. It is an iterative method starting from an initial point and converging to a local minimum. The function fmincon is a MATLAB implementation. The optimization processing of fmincon consists of three main stages:

(i) updating of the Hessian matrix of the Lagrangian function, (ii) quadratic programming problem solution, and (iii) line search and merit function calculation. This iteration is repeated until an optimal or feasible solution is found [22]. The local optimization method is applied with different starting points to the problem of evaluating a clearance criterion for the UAV obstacle avoidance systems.

This iteration is repeated until a specified termination criterion (either maximum number of function evaluations or convergence accuracy) is met. The results of the minimum distance to the obstacle and worst case parameters with different starting points are given in Table.1. The results clearly show that fmincon does not give the same solutions for this problem because the solution for a local optimization algorithm depends on the starting point. It does not give the true worst case. Therefore, global optimization methods are applied to find the true worstcase.

TABLE.1. LOCAL OPTIMIZATION RESULTS

\begin{tabular}{cccc}
\hline Algorithm & Starting point & Convergent point & $d_{\min }(m)$ \\
& {$\left[m, C_{m \delta e}, C_{\delta t}\right]$} & {$\left[m, C_{m \delta e}, C_{\delta t}\right]$} & \\
\hline fmincon & {$[\mathbf{1 . 5 2}, \mathbf{- 1 . 1 3}, \mathbf{1 1 . 8 2 4 3}]$} & {$[\mathbf{2 . 2 8 , - 1 . 2 4 3 , ~ 1 0 . 9 7 1 ]}$} & $\mathbf{2 9 . 0 2 3 5}$ \\
fmincon & {$[1.71,-1.13,13.409]$} & {$[2.28,-1.0193,10.971]$} & 27.4967
\end{tabular}




\section{Stochastic Global Optimization-based worst case analysis}

\section{A. Genetic Algorithms}

Genetic Algorithms (GA's) are general purpose stochastic search and optimization algorithms, based on genetic and evolutionary principles. The theory and practice of the GA was originally invented by John Holland in 1960s and was fully elaborated in his book Adaption in Natural and Artificial Systems published in 1975 [23]. The basic idea of the approach is to start with a set of designs, randomly generated using the allowable values for each design variable. Each design is also assigned a fitness value. The process is continued until a stopping criterion is satisfied or the number of iterations exceeds as a specified limit. Three genetic operators are used to accomplish this task: Selection, Crossover, and Mutation. Selection is an operator where an old design is copied into the new population according to the design's fitness. There are many different strategies to implement this selection operator including roulette wheel selection, tournament selection and stochastic universal sampling. The crossover operator corresponds to allowing selected members of the new population to exchange characteristics of their designs among themselves. Crossover entails selection of starting and ending positions on a pair of randomly selected strings, and simply exchanging the string of 0's and 1's between these positions. Mutation is the third step that safeguards the process from a complete premature loss of valuable genetic material during selection and crossover. The foregoing three steps are repeated for successive generations of the population until no further improvement in fitness is attainable [24, 25, 26].

GA can be applied to the UAV collision avoidance system to find the global minimum. The uncertain parameter set is considered here as the genetic representation, i.e. the chromosome. Each of the uncertainties corresponds to one gene. A binary coded string is generated to represent the chromosome, where each of the uncertain parameters lies between the lower and upper bounds. The selection function of roulette wheel is used for this study. The population size and crossover fraction are selected as default value of 20 and 0.8 respectively. The optimization is terminated after 51 iterations because the convergence accuracy is met. The GA results with different starting points are given in Table.2. Fig.5 shows the number of generations versus the best fitness and the mean fitness values at starting points [1.52, -1.13 , 11.82].

TABLE.2. GA RESULTS FOR A UAV OBSTACLE AVOIDANCE SYSTEM

\begin{tabular}{ccccccc}
\hline Algorithm & Starting point & $m(k g)$ & $\mathrm{C}_{\mathrm{m} \delta \mathrm{e}}$ & $\mathrm{C}_{\delta \mathrm{t}}$ & $d_{\min }(m)$ & Time \\
\hline$G A$ & {$[1.52,-1.13,11.82]$} & 2.2773 & -1.0283 & 10.9734 & 27.5018 & $12 \mathrm{sec}$ \\
\multirow{2}{*}{$G A$} & {$[1.71,-1.13,13.409]$} & 2.28 & -1.0177 & 10.9905 & 27.5032 & $12 \mathrm{sec}$ \\
& & & & & & \\
\hline
\end{tabular}




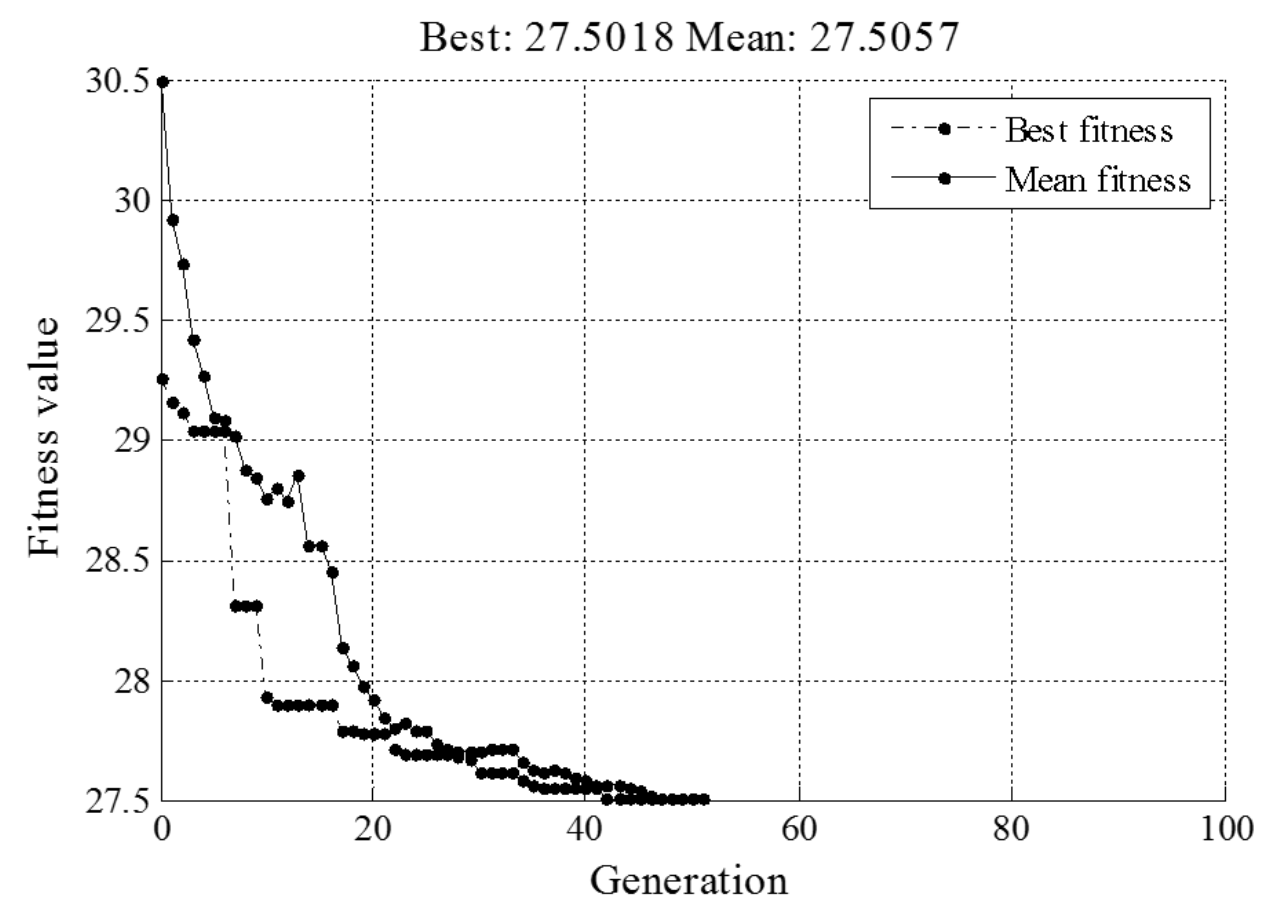

Fig.5. No of generations vs. Fitness value

\section{B. GLOBAL Algorithm}

The multistart clustering algorithm presented in this work is based on GLOBAL developed by Csendes in 1988, which is a modified version of the stochastic algorithm by Boender et al (1982) implemented in FORTRAN. The GLOBAL method has two phases i.e. a global and a local one. The global phase consists of sampling and clustering, while the local phase is based on local searches. A general clustering method starts with the generation of a uniform sample in the search space (the region defined by lower and upper bounds). After transforming the sample (by selecting a user set percentage of the sample points with the lowest function values), the clustering procedure is applied. Then, the local search is started from those points which have not been assigned to a cluster. GLOBAL uses the Single Linkage clustering rule [27].

The new implementation GLOBALm, which has been written in MATLAB, is freely available for academic purposes. It is the bound constrained global optimization problems with a black-box type objective function. GLOBALm has different local optimization methods which are capable of handling constraints. The UNIRANDI local search method is part of GLOBAL package while the BFGS (Broyden-Fletcher-Goldfarb-Shanno) local search is part of the MATLAB package. GLOBAL has six parameters to set: the number of sample points, the number of best points selected, the stopping criterion parameter for local search, the maximum number of function evaluations for local search, the maximum number of local minima to explore, and the used local method. All these parameters have a default value [27].

The GLOBAL optimization with UNIRANDI local search method is applied to find the global solution for the UAV obstacle avoidance system. The results with different numbers of the sampling points are given in Table.3. GLOBAL algorithm gives the nearly same solutions 
with different number of sampling points. It takes 1112 functions evaluation with 200 sampling points while 9810 functions evaluations with 500 sampling points. 36 local minimum are found at 500 sampling points while 8 found at 200 sampling points. GA and GLOBAL algorithms are performed well for this case study. However, both these algorithms cannot guarantee the worst case is found.

TABLE.3. GLOBAL RESULTS FOR UAV OBSTACLE AVOIDANCE SYSTEM

\begin{tabular}{ccccccc}
\hline No of SAMPLE & $m(k g)$ & $\mathrm{C}_{\mathrm{m} \delta \mathrm{e}}$ & $\mathrm{C}_{\delta \mathrm{t}}$ & $d_{\min }(m)$ & Fun.Evalu taken & Time \\
\hline 200 & 2.2063 & -1.0265 & 11.2221 & 27.748 & 1112 & 2 mins \\
500 & 2.1798 & -1.0171 & 10.971 & 27.494 & 9810 & 2 hours 15 mins \\
\hline
\end{tabular}

\section{Deterministic Global Optimization-based worst case analysis}

\section{A. DIRECT Method}

The disadvantage of the stochastic global optimization methods including GA and GLOBAL algorithms is that there are no formal proofs of convergence. In order to avoid this problem, a deterministic global optimization algorithm known as DIRECT method (DIviding RECTangles) is also considered in the verification process for the obstacle avoidance. The DIRECT algorithm was developed by Jones et al in 1993 [28], which guarantees to the convergence to the globally optimal if the objective function is continuous or at least continuous in the neighborhood of the global optimum. The global convergence may come at the expense of a large and exhaustive search over the domain. The DIRECT algorithm was created in order to solve difficult global optimization problems with bound constraints and a real-valued objective function. The DIRECT method does not require any derivative information. It is a modification of the standard Lipschitzian optimization method. This global search algorithm can be very useful when the objective function is a "black-box" function. The DIRECT algorithm is described below [24, 29, 30]

\section{Normalization and Division of the Hyper-cube}

DIRECT begins the optimization by transforming the domain of the problem into a unit hyper-cube. That is,

$$
\bar{\Omega}=\left\{X \in R^{N}: 0 \leq X_{i} \leq 1\right\}
$$

The algorithm works in this normalized space. Let $c_{l}$ be the center point of this hypercube and evaluate $f\left(c_{1}\right)$. The next step is to divide this hyper-cube by evaluating the function values at the points $c_{1} \pm \delta e_{i}, i=1,2, \ldots N$, where $\delta$ is one-third of the side length of the hyper-cube, 
and $e_{i}$ is the $i$ th unit vector. That is, a hyper-cube is divided into three hyper-rectangles in each dimension.

The DIRECT algorithm chooses to leave the best function values in the largest space; therefore, we define

$$
\boldsymbol{\omega}_{i}=\min \left(f\left(c_{1}+\delta e_{i}\right), f\left(c_{1}-\delta e_{i}\right)\right), 1<i<N
$$

and then divide the dimension with the smallest $\boldsymbol{\omega}_{i}$ into thirds, so that $c_{1} \pm \delta e_{i}, i=1,2, \ldots N$ are the centers of the new hyper-rectangles. This pattern is repeated for all dimensions on the "centre hyper-rectangle", choosing the next dimension by determining the next smallest $\boldsymbol{\omega}_{i}$.

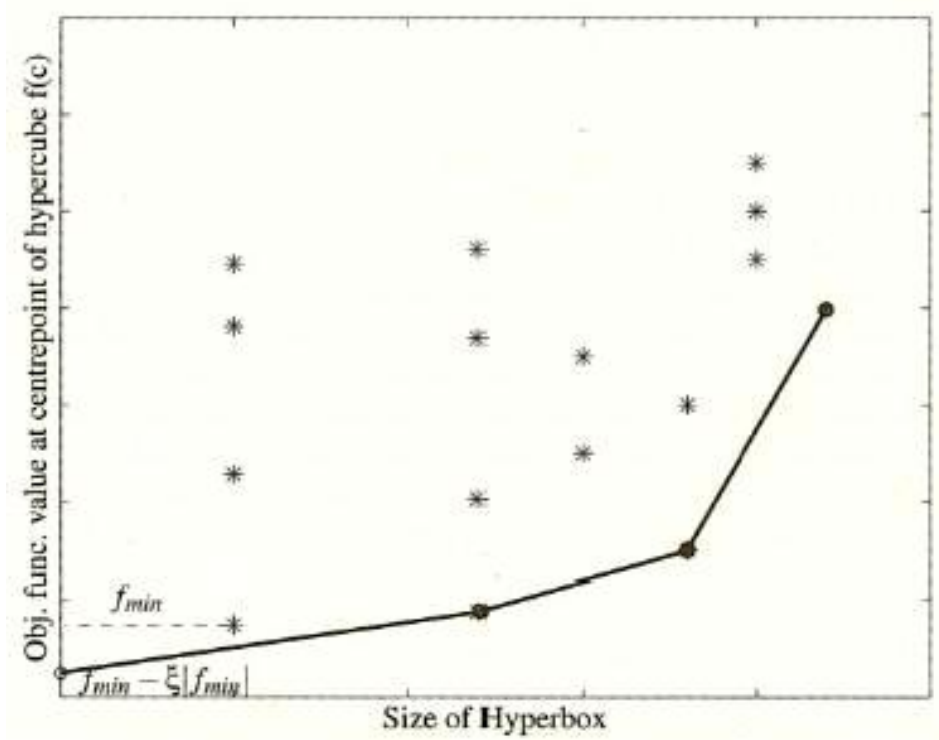

Fig.6. Hyper-rectangles on the piecewise linear curve are potentially optimal [24]

\section{Potentially Optimal Hyper-rectangles}

DIRECT then determines which rectangles are potentially optimal, and should be divided in this iteration.

Let $\epsilon>0$ be a positive constant and let $f_{\min }$ be the current best function value. A hyperrectangle $j$ is potentially optimal if there exists some $K>0$ such that

$$
\begin{gathered}
f\left(c_{j}\right)-K d_{j} \leq f\left(c_{i}\right)-K d_{i}, \forall_{i} \text { and } \\
f\left(c_{j}\right)-K d_{j} \leq f_{\text {min }}-\epsilon\left|f_{\text {min }}\right|
\end{gathered}
$$

In (18), $c_{j}$ is the center point of the hyper-rectangle $j$, and $d_{j}$ defines a measure for the hyperrectangles. Jones et al. chose to use the distance from center point $c_{j}$ to its vertices as the measure and also concluded that a good value for $\epsilon$ is $1 \times 10^{-4}$. Fig.6 illustrates this definition. 


\section{Division of the Hyper-rectangles}

Once a hyper-rectangle has been identified as potentially containing the optimal solution, DIRECT divides this hyper-rectangle into smaller hyper-rectangles. DIRECT divides the hyper-rectangles by performing division only in the dimensions with the longest side length. The sequence of the dimensions to be divided is determined by $\boldsymbol{\omega}_{j}$ which is defined as

$$
\omega_{j}=\min \left(f\left(c_{i}+\delta_{i} e_{j}\right), f\left(c_{i}-\delta_{i} e_{j}\right)\right), \quad j \in I
$$

where $\delta_{i}$ is one-third the length of the longest side of hyper-rectangle $I, e_{j}$ is the $j$ th unit vector, and $I$ is the set of all dimensions of the longest side length. This process is repeated for all dimensions in $I$.

\section{B. Simulation Results}

The DIRECT algorithm is applied to the UAV obstacle avoidance verification process, and the results are given in Table.4. The DIRECT method requires no initial guesses but operates on the parameters upper and lower bounds. The DIRECT algorithm terminates as soon as it exceeds the given iterations. The history of iteration versus fitness value is shown in Fig.7. All optimization algorithms are performed in MATLAB 2010a and Intel (R) Core(TM) 2 Duo CPU (3.16GHz). DIRECT takes 3 hours 35 minutes to converge to the global minimum. Compared to the stochastic global algorithms, GA and GLOBAL algorithm are performed well for this case study, but GA performs faster. However, these are stochastic global algorithms and there is no confidence to establish the true worst case. The DIRECT algorithm can guarantee finding the worst case in this application, but the computation time is high.

These worst-case condition and worst-case parameters identified in the verification process are further validated with simulation response shown in Fig.8. The time versus distance to the obstacle at the nominal and worst-case parameters is shown in Fig.9. The worst-case minimum distance to the obstacle $d_{\min }$ is $27.4982 \mathrm{~m}$ which is greater than the specified safety radius of the obstacle. This concludes that the obstacle avoidance algorithm and the controller provide adequate performance at the worst-case parameters. Furthermore, in the presence of all the described variations, the safety margin for anti-collision is respected.

TABLE.4. DIRECT RESULTS FOR A UAV OBSTACLE AVOIDANCE SYSTEM

\begin{tabular}{lccccccc}
\hline Algorithm & Iteration & $m(k g)$ & $\mathrm{C}_{\mathrm{m} \delta \mathrm{e}}$ & $\mathrm{C}_{\delta \mathrm{t}}$ & $d_{\min }(m)$ & Fun.Evalu taken & Time \\
\hline DIRECT & 500 & 2.28 & -1.017 & 10.976 & 27.498 & 18505 & $3 \mathrm{~h} 35 \mathrm{~m}$ \\
\hline
\end{tabular}




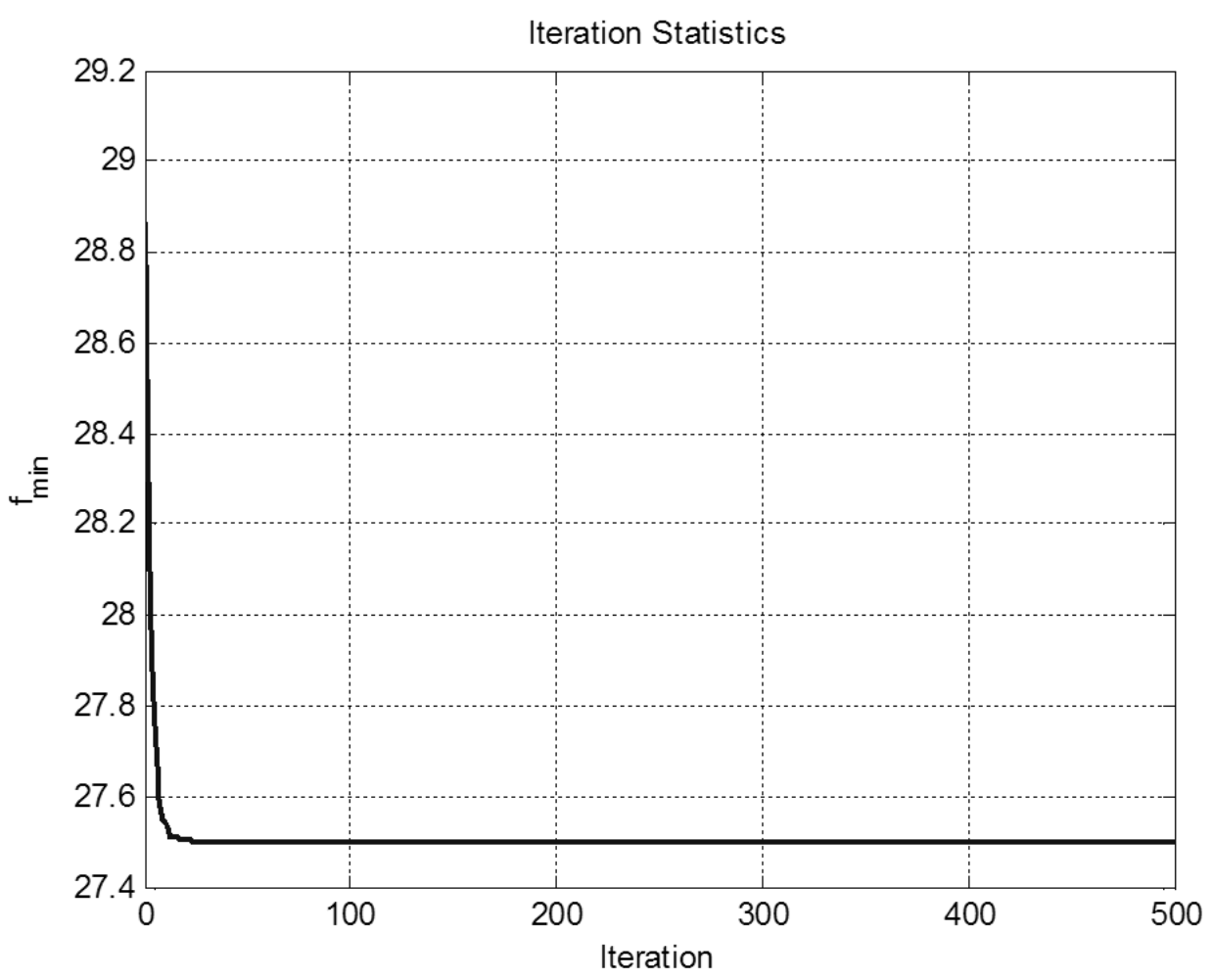

Fig.7. DIRECT algorithm- Iteration vs. Fitness value

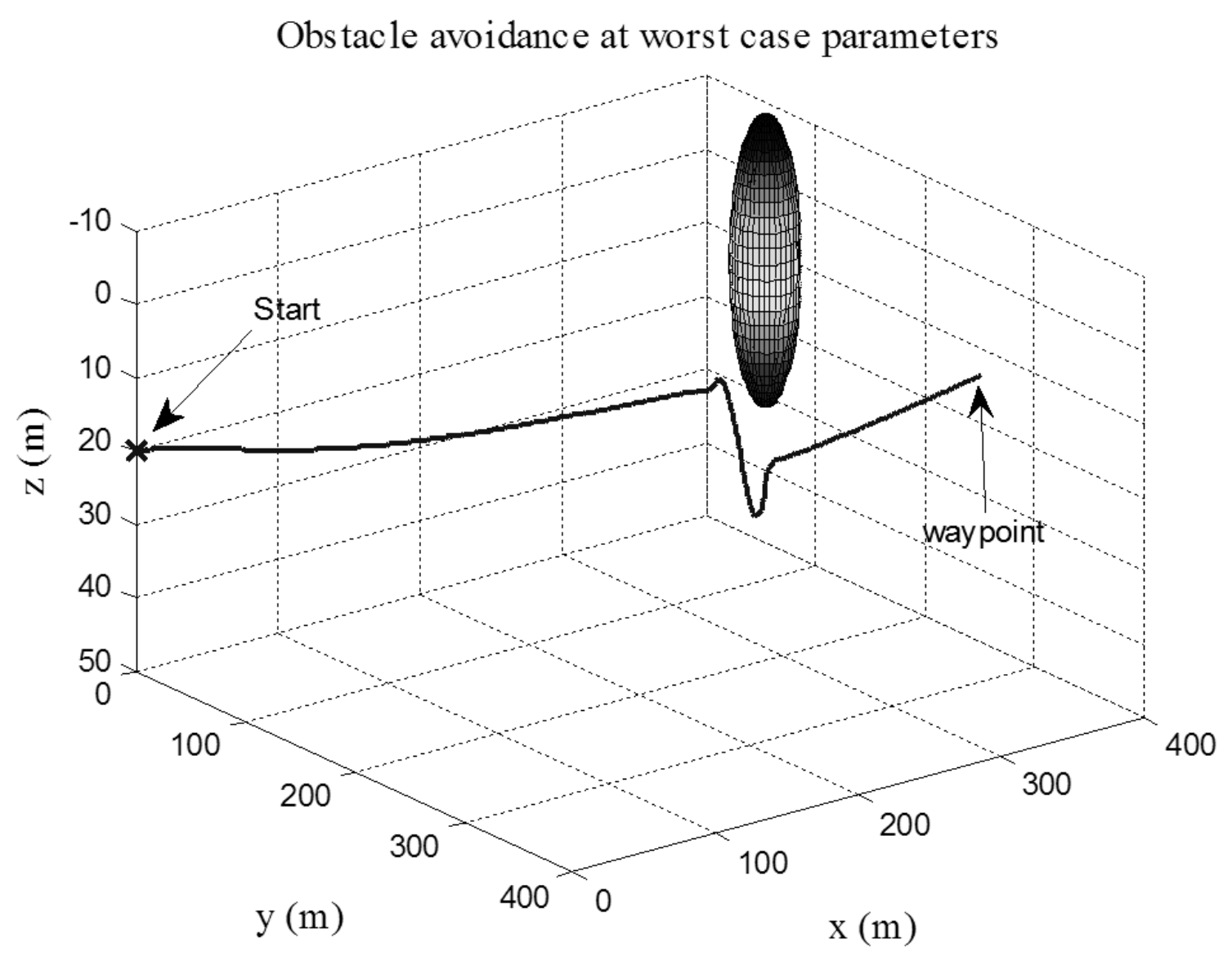

Fig.8. Simulation response at worst-case parameters 


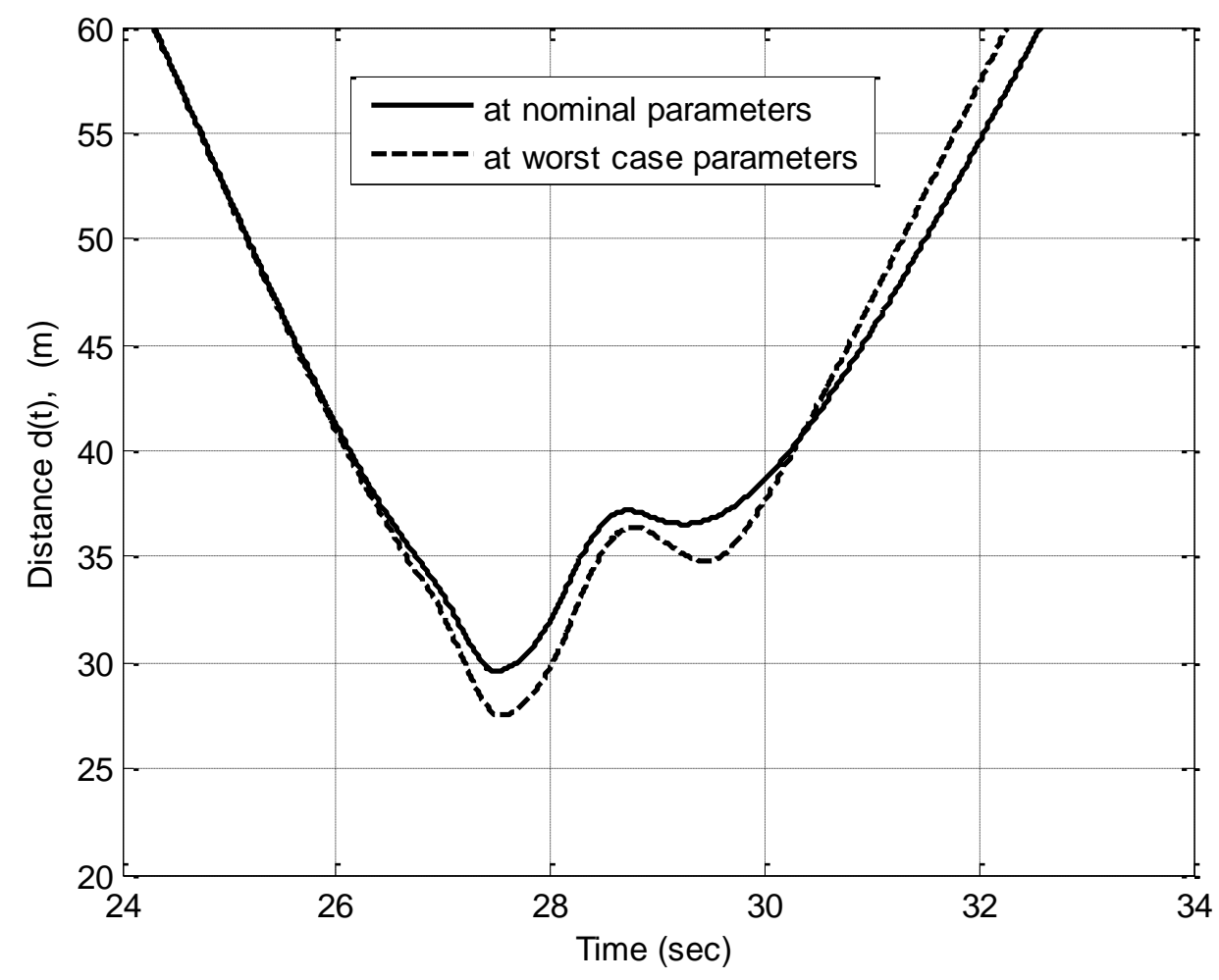

Fig.9. Time vs distance to the obstacle at nominal and worst case parameters

\section{Conclusions}

In this paper, optimization based clearance process of obstacle avoidance systems is applied to verify collision avoidance algorithms for UAVs. The key idea in this verification approach is that it is not necessary for an optimization algorithm to evaluate a cost function over all possible solutions in order to find the optimal solution. However different from many optimization problems, it is important to find all the possible worst cases in order to verify safety critical functionalities like obstacle avoidance. This requires an optimization algorithm that converges to the global optimal solution.

In developing optimization based worst case analysis for verification of collision avoidance algorithms, the minimum distance to the obstacle during collision avoidance maneuver is defined as the cost function. To demonstrate the concept, a 6DOF UAV model is used in the case study with a designed collision avoidance algorithm, and mass and two aerodynamic coefficients variations are considered for the verification purpose. The local optimization method does not give a unique solution as different worst cases are identified when the optimization starts from different initial conditions. Therefore, the local optimization is not suitable for verification of collision avoidance algorithms for this case study.

To overcome this problem, global optimization algorithms are studied. Stochastic global optimization algorithms including GA and GLOBAL methods have been applied to the problem. GA and GLOBAL algorithm perform well for this UAV problem. The deterministic global optimization of the DIRECT method has also been investigated for the worst-case 
analysis. Compared with other global optimization algorithms in this study, the DIRECT algorithm can guarantee finding the worst case, although it takes more time to converge. It shall be highlighted that in developing collision avoidance algorithms using the potential field method, the UAV is considered as a mass point and only the kinematic model is used. However in real implementation of collision avoidance maneuver, the UAV has to be controlled to follow the desirable total velocity and attitude. Therefore, the UAV dynamics and the influence of the inner loop controllers for tracking reference speed and attitude provided by the collision avoidance algorithm must be taken into account in order to fully understand the behavior of the collision avoidance algorithm. This is particularly important for very close maneuvers like collision avoidance. The work presented in this paper provides a framework of taking into account the different levels of the model complexity used in the different stages of autonomous control development. It can significantly improve the efficiency of the verification process by automatically searching the worst cases without the need to exhaustively evaluate all possible combinations of variations. Further work will be on applying the proposed verification approach to an environment with dynamic obstacles for UAVs.

\section{References}

[1] C. Fielding, A. Varga, S. Bennai, M. Selier(Eds). Advanced techniques for clearance of flight control laws. Springer LNCIS.283, pp. 3-33, 107-117, Heidelberg 2002.

[2] B. Cheol Min, H. Y. Kwon, D. Kim. Path planning algorithm for VTOL type UAVs based on the methods of ray tracing and limit cycle. Computational Intelligence in Robotics and Automation (CIRA), IEEE International Symposium, pp. 296-301, 15-18 Dec 2009.

[3] S. Griffiths, J. Saunders, A. Curtis, T. McLain. Obstacle and terrain avoidance for miniature aerial vehicles. IEEE Robotics and Automation Magazine, Vol-13, No-3, pp. 3443, September 2006.

[4] P. O. Pettersson, P. Doherty. Probabilistic roadmap based path planning for an autonomous unmanned helicopter. Journal of Intelligent and Fuzzy systems, Vol-17, No-4, pp. 395-405, September, 2006.

[5] S. A. Bortoff. Path planning for UAVs. Proceedings of the American Control Conference, Vol-1, pp. 364-368, June 2000.

[6] T. Lapp, L. Singh. Model predictive control based trajectory optimization for nap-ofthe-earth (NOE) flight including obstacle avoidance. Proceeding of the 2004 American Control Conference, Boston, Massachusetts, June 30-July 22004.

[7] G. P. Roussos, D. V. Dimarogonas, K. J. Kyriakopoulos, 3D navigation and collision avoidance for a non-holonomic vehicle. American Control Conference 2008, Washington, USA, June 11-13, 2008. 
[8] T. Paul, T. R. Krogstad, J. T. Gravdahl. UAV formation flight using 3D potential field. Control and Automation 2008, $16^{\text {th }}$ Mediterranean Conference on. pp. 1240-1245. 25-27 June 2008.

[9] K.K. James. Safety analysis methodology for unmanned aerial vehicle (UAV) collision avoidance systems, MIT Lincoln Laboratory, Lexington, MA, United States Air Force, \#F19628-00-C-0002.

[10] N. Snooke, C. Price, C. Downes, C. Aspey. Automated failure effect analysis for PHM of UAV. Proceedings of the International System Safety and Reliability Conference (ISSRC 2008), ISBN: 978-981-08-0446-6, April 2008.

[11] E. W. Roland, R. John Hansman. Safety considerations for operation of unmanned aerial vehicles in the national airspace system. MIT International Center for Air Transportation, Massachusetts Institute of Technology, Cambridge, USA, March 2005.

[12] R. Lutz, S. Nelson, A. Patterson-Hine, C. R. Frost, D. Tal. Identifying contingency requirements using obstacle analysis. Proceedings $13^{\text {th }}$ IEEE International Conference on Requirements Engineering, pp- 263-272, 29 Aug- 2 Sept 2005.

[13] S. Temizer, M. J. Kochenderfer, L. P. Kaelbing, T. L. Perez, J. K. Kuchar, Collision Avoidance for Unmanned Aircraft using Markov Decision Processes, AIAA Guidance, Navigation, and Control Conference, Toronto, Ontario, Aug 2-5, 2010.

[14] T. Schouwenaars, J. How, Eric Feron, Decentralized cooperative trajectory planning of multiple aircraft with hard safety guarantees, AIAA Guidance, Navigation, and Control Conference and Exhibit, 16-19 August 2004, Providence, Rhode Island.

[15] S. Srikanthakumar, W. H. Chen. Optimisation-based clearance process for obstacle avoidance systems. International Conference on Automation and Computing, Birmingham, $11^{\text {th }}$ September 2010.

[16] R. Carona, A. P. Aguiar, J. Gaspar. Control of unicycle type robots tracking, path following and point stabilization. International Proceeding of IV Electronics and Telecommunications, pp. 180-185, Lisbon, Portugal, November 2008.

[17] A. D. Luca, G. Oriolo. Local Incremental Planning for Nonholonomic Mobile Robots. Proceeding.of.IEEE International Conference on Robotics and Automation, San Diego, CA, pp. 104-110, 8-13 May 1994.

[18] Y. C. Paw. Synthesis and validation of Flight control for UAV, A dissertation, The faculty of the graduate school, The university of Minnesota, December 2009.

[19] O. Khatib, Real-time obstacle avoidance for manipulators and mobile robots, International Journal Robotics Research, Vol.5, N0-1, pp, 90-99, 1986. 
[20] Mario Isabel Ribeiro, Obstacle avoidance, Instituto de Sistemas e Robotica, Nov, 2005. http://users.isr.ist.utl.pt/ mir/

[21] Eliana Costa e Silva, E. Bicho, W. Erlhagen, The potential field method and the nonlinear attractor dynamics approach: what are the difference?, 7th Portuguese Conference on Automatic Control", CONTROLO 2006.

[22] Mathworks.co.uk

[23] J. Holland, Adaption in natural and artificial Systems, University of Michigan press, Ann Arbor, MI 1975.

[24] D. Bates, M. Hagstrom, Nonlinear Analysis and Synthesis Techniques for Aircraft Control, Springer, pp. 259-300, 2007.

[25] J. S. Arora, Introduction to optimum design, second edition, Elsevier, Academic Press.

[26] W. M. Rand, Controlled observations of the Genetic algorithm in a changing environment: case studies using the Shaky ladder hyperplane-defined functions, Thesis, The University of Michigan, 2005.

[27] Tiber Csendes et al, The GLOBAL Optimisation Method Revisited, University of Szeged, Hungary, September, 2008.

[28] D. Jones, DIRECT Global optimization algorithm, Encyclopedi of Optimization, Kluwer Academic Publishers, 2001.

[29] D. E. Finkel, C. T. Kelley, Convergence Analysis of the Direct Algorithm, North Carolina State University, July, 2004.

[30] D. E. Finkel, DIRECT optimization algorithm user guide, Center for Research in Scientific Computation, North Carolina State University, March 2, 2003. 